\title{
How Professionals Tinker With Ethical Tensions Collectively When Fostering Service User Autonomy: An Ethnographic Study In Long-term Care
}

\section{Marjolijn Heerings ( $\nabla$ heerings@eshpm.eur.nl )}

Erasmus Universiteit Rotterdam https://orcid.org/0000-0002-8280-0305

Hester van de Bovenkamp

Erasmus Universiteit Rotterdam

Mieke Cardol

Hogeschool Rotterdam

Roland Bal

Erasmus Universiteit Rotterdam

\section{Research article}

Keywords: Quality of care, professional-patient relations, intellectual disability, psychiatric rehabilitation, mental health recovery, long-term care, social work, community participation, ethics, autonomy

Posted Date: August 11th, 2020

DOI: https://doi.org/10.21203/rs.3.rs-36856/v2

License: (c) (i) This work is licensed under a Creative Commons Attribution 4.0 International License.

Read Full License 
The authors have withdrawn this preprint from Research Square 